\title{
New treatments for Tuberculosis
}

Diacon et al report a promising advance in both the future management of tuberculosis (TB) and the future design of drug trials in this field. ${ }^{1}$ The authors undertook a prospective, randomised study in uncomplicated, treatment naive patients with smear-positive pulmonary TB in South Africa.

The study assessed the early bactericidal activity (EBA) of novel combinations of new and existing drugs, but avoiding rifampicin and isoniazid, over the first 14 days of treatment, using the novel outcome measure of daily fall in colony forming units (CFUs) of Mycobacterium tuberculosis per millilitres of sputum. This approach has the potential to expedite the assessment of new individual drugs and combination regimens.

The regimen of PA-824-moxifloxacin-pyrazinamide demonstrated significantly higher EBA at day 14 than that of bedaquiline, bedaquiline-pyrazinamide and bedaquiline-PA- 824 , but not PA-824-pyrazinamide and, while underpowered to demonstrate a significant difference, comparable with the standard regimen of rifampicin, isoniazid, pyrazinamide and ethambutol. Additionally, this regimen appears to produce a more rapid fall in CFUs than the majority of the other regimens tested.

This study should be interpreted with cautious optimism at this stage; this regimen requires further investigation over the full course of treatment to ensure the promising results at 14 days persist and to ascertain the optimum duration of treatment. Additionally, further assessment of the risk of adverse reactions is required. However, if the early promise of this regimen is confirmed, it would provide the first entirely new regimen for many years. The avoidance of rifampicin reduces the likelihood of drug interactions. However, the key advantage of this regimen is the current absence of resistance to these new drugs, but these drugs must be used judiciously to preserve this status.

- AH Diacon, R Dawson, F von Groote-Bidlingmaier, et al. 14-day bactericidal activity of PA-824, bedaquiline, pyrazinamide, and moxifloxacin combinations: a randomised trial. Lancet 2012;380:986-93. 